\title{
Einleitung in den Schwerpunkt
}

\begin{abstract}
Nur wer glaubt, die theoretische Empirie der Neuzeit sei so etwas wie die <natürlichste Sache von der Welt>, die nur freizulegende und endlich zugelassene Unmittelbarkeit zu den Dingen, kann es für rhetorische Redundanz halten, wenn dieses Angebot in der Sprache der Rivalität mit dem Buch, der Überbietung aller vorherigen Lesbarkeiten erfolgte.
\end{abstract}

HANS BLUMENBERG, Die Lesbarkeit der Welt, I979

Für Geistes- und Kulturwissenschaften ist es kein besonders neuer Sachverhalt, dass ihre Methoden, ihre Wissensformen und Erkenntnisse dem Verdacht ausgesetzt sind, dem Bereich der bloßen Spekulation anzugehören und irgendwie weniger stichhaltig zu sein als die der empirisch verfahrenden Natur-, aber auch der Sozialwissenschaften. Verlegt man sich nicht von vornherein auf den Hinweis, dass die kritische, methodisch reflektierte Analyse und Auslegung von Texten, Filmen und Tonaufzeichnungen eine der Rechtfertigung nicht weiter bedürftige, eigenständige Form der Empirie darstellt, dann bleiben den Geistes- und Kulturwissenschaften auf den ersten Blick nur zwei alternative Strategien, um ihren Legimitationsstress zu lindern: Entweder wird die Distanz zur Empirie als konstitutive Besonderheit gefeiert, die den Geltungsanspruch der Geistes- und Kulturwissenschaften gerade ausmacht; oder man akzeptiert die Dominanz und den vermeintlichen epistemologischen Vorrang der Empirie und stellt die eigene Forschung unter den Vorbehalt einer weiteren Überprüfbarkeit im Rahmen quantitativer oder naturwissenschaftlich-exakter Verfahren.

In der Medienwissenschaft zeigt sich diese Ambivalenz gegenwärtig besonders deutlich: Auf der einen Seite gründet die Medienwissenschaft als kulturwissenschaftliche Disziplin ihr Selbstverständnis nach wie vor auf den Kontrast mit der Kommunikationswissenschaft, die teilweise verwandte Gegenstände untersucht, dies aber eben vorzugsweise mit den Methoden einer 
sozialwissenschaftlichen Empirie. Auf der anderen Seite nimmt die Anzahl der Forschungsprojekte zu, die sich etwa durch Kooperation mit der Kognitionspsychologie oder der Neurowissenschaft verbindliche Antworten auf schon lange diskutierte medienästhetische Fragestellungen versprechen.

Gerade mit den ihr eigenen Gegenstandsbereichen, Methoden und Theorien sowie aus ihrer Fachgeschichte heraus hat die Medienwissenschaft aber auch die Möglichkeit, diese vereinfachende Dichotomie zwischen empirischen und nicht-empirischen Verfahren zu unterlaufen und die Frage, was Empirie und welches Wissen denn <empirisch> sei, neu zu perspektivieren.

Zum einen sind Entstehung und Erfolg der natur- und sozialwissenschaftlichen Empirie eng an mediale Möglichkeitsbedingungen gebunden. Wie jüngst nochmals von Adrian Johns mit seiner Rekonstruktion der Ko-Emergenz von Buchdruck, Copyright und moderner naturwissenschaftlicher Praxis im I7. Jahrhundert und von Mario Biagioli mit seiner Studie zu den technischen Vorrichtungen Galileis und ihrem Beitrag zu der Durchsetzung eines modernen Wissenschaftsverständnisses gezeigt, sind Medien in vielfältiger und historisch variierender Weise in die Ausbildung empirischer Verfahren unterschiedlicher Disziplinen involviert. ${ }^{1}$ Auch die moderne Konzeption von <Objektivität erweist sich im Licht der neueren Wissenschaftsforschung als abhängig von medialen Verfahren, die den Ausschluss von menschlicher <Subjektivität> sicherstellen. ${ }^{2}$ In diesen, wie in vielen anderen Studien aus dem Umfeld der Wissenschaftsforschung, geht es nicht einfach darum, die Empirie als genauso $<$ konstruiert> und <willkürlich> erscheinen zu lassen, wie es der dominante Diskurs mit den kulturwissenschaftlichen Verfahren tut; viel eher geht es darum, die Heterogenität und Historizität von Wissensformen zu rekonstruieren und die jeweils unvermeidlichen Verschränkungen von apparativen Funktionsweisen mit ästhetischen Kategorien nachzuvollziehen, ein Zusammenhang, dem

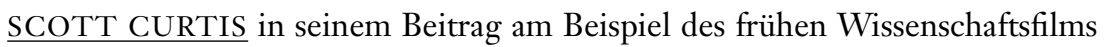
nachgeht.

Zum anderen legt aber auch die Geschichte medienwissenschaftlicher Forschungen selbst, nicht zuletzt aufgrund der Tatsache, dass Medienwissenschaft aus einer Reihe von Disziplinen und aus der Zusammenführung unterschiedlicher Methoden hervorgegangen ist, eine Neuperspektivierung von Empirie im Allgemeinen und ihres Status für die Konsolidierung eines Fachs Medienwissenschaft im Besonderen nahe. Wie die Beiträge von ISABELL OTTO und JOSH SHEPPERD zeigen, gilt für so gegensätzliche Bezugsfiguren der Medienwissenschaft wie Theodor W. Adorno und Marshall McLuhan gleichermaßen, dass ihre Theoretisierung von Medien in einem ebenso unmittelbaren wie spannungsvollen Bezug zur empirischen Kommunikationsforschung steht. In beiden Fällen (und dies mag für Adorno überraschender erscheinen als für McLuhan) führt nicht zuletzt das Interesse an der spezifischen Materialität - und allgemeiner: der Medialität - der Medien zu einem konzeptuellen und methodischen Konflikt mit den empirischen Projekten und Methoden, an denen sie jeweils
1 Adrian Johns, The Nature of the Book: Print and Knowledge in the Making, Chicago, III (University of Chicago Press) 1998; Mario Biagioli, Galileo's Instruments of Credit: Telescopes, Images, Secrecy, Chicago, III (University of Chicago Press) 2006. 2 Lorraine Daston, Peter Galison, Objectivity, New York (Zone Books) 2007. 
beteiligt waren. Dieser Konflikt kann indes nicht auf eine schlichte Zurückweisung von Empirie reduziert werden, entwickeln doch Adorno wie McLuhan ihre genuin eigenen Verfahren, mit denen sie die <versteckten>, einer schlichten Befragung oder Programmanalyse nicht zugänglichen Wirksamkeiten von Medien entziffern wollen.

Soweit es sich dabei um Gründungsmomente medienwissenschaftlichen Forschens handelt, liegt ihre Signifikanz also nicht zuletzt darin, dass in beiden Fällen die Frage nach dem Gegenstand <Medien> und der ihm angemessenen Empirie virulent wurde. Wenn in den unterschiedlichen Ansätzen der I97oer und I98oer Jahre - durchaus im Geist, wenn auch nicht zwingend in direkter Nachfolge von McLuhan und Adorno - die kulturellen Praktiken, die textuellen Formen oder die apparativen Konstellationen in den Fokus geraten, dann ist dies jeweils auch mit dem Anspruch verbunden, eine Art Gegen-Empirie zu entwerfen, die über die Gleichsetzung von Empirie mit Vermessen und Befragen hinausreicht. Und wenn JAN-HENDRIK BAKELS und HERMANN KAPPELHOFF in ihrem Beitrag zu diesem Heft ein neues Modell der empirisch-psychologischen Operationalisierung film- und medienästhetischer Fragestellungen entwickeln, dann tun sie dies gerade aus dem Bestreben heraus, die von einer vielleicht allzu selbstgewiss als empirisch auftretenden Medienwirkungsforschung nicht erfassten und in der Regel noch nicht einmal bedachten Dimensionen der Medienästhetik in den Blick zu rücken.

Am explizitesten wird diese Methodendiskussion bislang indes in den Cultural Studies geführt. Diese setzen zwar häufig das sozialwissenschaftliche Instrumentarium ein, wenn auch meist <nur〉 das qualitativer Empirie. Gegen eine vermeintliche Gleichsetzung von Cultural Studies und Rezeptionsforschung hat Ien Ang allerdings deutlich gemacht, dass das Interesse an «complex structural and conjunctural determinations of viewership and audiencehood $»^{3}$ nicht einfach nur die Ergänzung der Empirie durch Theorie erfordert, sondern eine andere Empirie, die Texte und Praktiken auf einer gemeinsamen Ebene behandelt.

In ähnlicher Weise hat die <New Film History> mit ihrer Umorientierung vom Film auf das Kino und mit der darauf folgenden Erweiterung des Untersuchungshorizonts über den kanonischen Autorenfilm hinaus hin zu den audiovisuellen Bildpraktiken und ihren institutionellen und diskursiven Rahmungen den Horizont dessen drastisch erweitert, was die Medienwissenschaft als ihre Untersuchungsgegenstände ansieht. ${ }^{4}$ Die Integration wirtschafts- und technikhistorischer sowie sozialwissenschaftlicher Ansätze in den r970er und I980er

3 len Ang, On the Politics of Empirical Audience Research, in: Living Room Wars. Rethinking Audiences for a Postmodern World, London/ New York (Routledge) 1996, 35-52, hier 41.

4 Paul Kusters, New Film History. Grundzüge einer neuen Filmgeschichtswissenschaft, in: montage/av 5,1 1996, 39-6o. Jahren und die Entwicklung neuer Ansätze wie der Medienarchäologie seit den I990er Jahre haben dabei nicht nur das Feld der Gegenstände der Medienhistoriografie erweitert, sondern auch deren Bestimmung und Kontur und die zu ihrer Untersuchung erforderlichen Ansätze und Methoden.

Am drastischsten zeigt sich der Anspruch, mit einer Neudefinition dessen, was Medien ausmacht, auch das Forschungsinstrumentarium zu verändern, sicherlich in den verschiedenen medienarchäologischen und technikanalytischen 
Ansätzen, die schon durch ihr Interesse an Schaltplänen, Informationsverarbeitung, Physiologie etc. ihren Erkenntnissen die Autorität empirischer Fundierung verleihen, ohne dabei einem etablierten Modell von Empirie einfach zu folgen.

Diese fortlaufende Neuperspektivierung der Empirie durch die Medienwissenschaft setzt sich gegenwärtig auch deshalb fort, weil die technischen Medien, die weiterhin ihren zentralen Gegenstandsbereich bilden, zunehmend selbst mithilfe technischer Medien untersucht werden. Es gilt fortan für die Medienwissenschaft nicht weniger als etwa für die Naturwissenschaften, dass ihr Gegenstand nicht nur als Medium, sondern in Medien bestimmt wird und dass seine empirische Erschließung medienbasiert erfolgt. Dies ist zunächst keine besonders überraschende Einsicht. Schon Bibliothek und Wunderkammer, Museum und Camera Obscura lassen sich als konstitutive Instrumentarien humanwissenschaftlichen Wissens betrachten; auch die Konsequenz etwa des Dia-Projektors für die empirische Arbeit am Material der Kunstgeschichte ist einschlägig. ${ }^{5}$ Die gegenwärtig exponentiell zunehmende Möglichkeit der Verdatung wirft allerdings wiederum ganz neue Fragen zur empirischen Erfassung des Gegenstands und zum Verhältnis der so gewonnenen Daten zu politischen, ästhetischen und philosophischen Konzepten auf. So stellt die Möglichkeit der computerbasierten Erfassung und der seriellen Analyse großer Werkkorpora gerade die Filmwissenschaft vor die Herausforderung, ihren Gegenstand neu zu denken. Dabei eröffnet sich, wie BARBARA FLÜCKIGER in ihrem Beitrag anhand des filmischen Parameters der Farbe zeigt, die Möglichkeit, den Film vollends jenseits von Künstlerbiografien und ikonologisch inspirierten oder an Fragen der Repräsentation orientierten Einzelwerkanalysen als kulturelle Sequenz aufzufassen, d.h. als nur in großen Datensätzen fassbare, offene Abfolge diskursiv präformierter Lösungen für ästhetische Probleme, wie dies George Kubler vor fünf Jahrzehnten in The Shape of Things ${ }^{6}$ - noch ohne über die geeigneten Instrumentarien wirklich zu verfügen - einmal für die bildende Kunst vorgeschlagen hatte.

Eine weitere Pointe erhält die Frage nach der Medialität der Gegenstände und Methoden der Medienwissenschaft, wenn man bedenkt, dass die Medienforschung nicht notwendiger Weise ein Medium erst verdaten muss, um es empirisch zugänglich zu machen: In vielerlei Hinsicht sind die Forschungsgegenstände durch Verdatung konstituiert - durch technische Blaupausen, durch ökonomische Kalkulationen, vor allem aber durch umfassende Markt- und Zuschauer-/Nutzerforschung. Hier stellt sich erneut die Frage, ob und wie die Medienwissenschaft auf Distanz zu diesen Formen empirischer Verdatung gehen muss und ob und wie sie die medienkonstitutive Dynamik dieser Verdatungen (dennoch) in den Blick nehmen kann.

Mit dem Internet und mehr noch dem Web 2.o ergibt sich hier eine nochmals neue Situation, insofern viele der verdatenden und vermessenden Verfahren den täglichen Umgang mit dem Medium direkt strukturieren.
5 Heinrich Dilly, Lichtbildprojektion - Prothese der Kunstbetrachtung, in: Irene Below (Hg.), Kunstwissenschaft und Kunstvermittlung, Wien (Anabas) 1975, 153-172.

6 George Kubler, The Shape of Time: Remarks on the History of Things, New Haven, Conn. (Yale University Press) 1962. 
RICHARD ROGERS betrachtet es in seinem Beitrag deshalb als zentrale Aufgabe der Medienwissenschaft, diese das Internet kennzeichnenden Verfahrensweisen zu verstehen und schließlich für die eigene Forschung zu entwenden. Damit wird einerseits eine explizit medienspezifische Empirie eingefordert; andererseits wird das Internet damit aber auch zu einem vielfältigen und gestaltbaren Instrumentarium für die empirische Kultur- und Gesellschaftsanalyse, wobei auch dies wieder eine Neuperspektivierung des Empirischen impliziert, insofern es beispielsweise von demografischen zu post-demografischen Kategorien wechselt.

Auch dadurch, und in seinem Zusammenspiel mit den anderen Beiträgen zu diesem Heft, belegt Rogers' Beitrag in exemplarischer Weise, dass die Frage nach der Empirie immer wieder neu gestellt werden muss - gerade von der Medienwissenschaft und aus der Medienwissenschaft heraus.

VINZENZ HEDIGER, MARKUS STAUFF 